Pacific Journal of Mathematics

A NOTE ON EXPONENTIALS OF DISTRIBUTIONS 


\section{A NOTE ON EXPONENTIALS OF DISTRIBUTIONS}

\section{Alan SLOAN}

Nonstandard analysis is used to discuss nonlinear functions of distributions. An application is given to obtain a generalized Trotter product formula. The strong resolvent topology is discussed from a nonstandard point of view.

Enlarging the real number system to include infinite and infinitesimal quantities enabled Laugwitz [5] to view the delta function distribution as a point function. Independently Robinson [7] demonstrated that distributions could be viewed as generalized polynomials. Luxemburg [6] presented an alternate picture of distributions as generalized functions within the context of Robinson's theory of nonstandard analysis and it is a special case of this point of view that we take here. Once one accepts distributions as generalized functions, the composition map provides a natural method of defining nonlinear functions of distributions. Unfortunately, the difficulties in the standard attempt to define a function, $f$, of a distribution, $\tau$, (by first writing $\tau$ as a limit, in some appropriate sense, of smooth standard functions $\tau_{n}$, next composing these approximations with $f$, and finally taking the limit of $f \circ \tau_{n}$ ) still remain in the nonstandard theory. In particular, this procedure may not lead to a distribution and even when it does the distribution obtained may depend on the representation of the original distribution as a generalized function. Thus, at present, there is no comprehensive theory of nonlinear functions of distributions.

The development of such a theory may well proceed along alternate tracks depending on the applications intended. One use for distributions occurs in the study of perturbations of selfadjoint operators in Hilbert space. In some perturbation problems the pathology of obtaining compositions which do not represent distributions may be avoided by considering only bounded functions of distributions. For example, in the Trotter product formula one works with bounded exponentials. Even though, in this case, the exponentials of distributions may be identified with standard distributions it is the conclusion of this paper that such an identification should not be made. It is the author's view that functions of distributions should be regarded as generalized functions but not distributions. The utility of this view is illustrated by a nonstandard version of the Trotter product formula.

For an introduction to nonstandard analysis and its relation to distributions, see [10]. In [11] a square root for the delta function 
was defined nonstandardly.

Let ${ }^{*} X$ be a $\delta$-incomplete ultrapower of a structure $X$ containing the real numbers, $\boldsymbol{R}$. We shall use the convention that $A \subset^{*} A$, for all sets $A$ considered. If $T$ is a topological space with $u$ in $T$ and $v$ in ${ }^{*} T$ then we shall write $u \approx v$ if and only if $v$ is in $\cap\left\{\mathscr{U}_{b}: \mathscr{C}\right.$ is an open set containing $\left.u\right\}$. In this case we say $u$ is the standard part of $v$, relative to the given topology and that $v$ is near standard. We write $u=s t(v)$. We always assume that $\boldsymbol{R}^{p}$ has the Euclidean topology and all Hilbert spaces have the norm topology.

If $f$ and $g$ are Lebesgue measurable on ${ }^{*} S$ for some standard set $S$ which is measurable, we shall write $\langle f, g\rangle$ for $\int_{* S} f g d x$, providing the integral exists. If, in fact, $f$ and $g$ are standard functions then $\langle f, g\rangle=\int_{S} f g d x$, by the transfer principle.

Let $W$ be an open subset of $\boldsymbol{R}^{p}$. Let $B_{n}$ be an increasing sequence of relatively compact subsets of $W$ whose union is $W$ and whose closures are in $W$. If $\mathscr{D}=\bigcup_{n=1}^{\infty} C_{c}^{\infty}\left(B_{n}\right)$ is given the inductive limit topology then the elements of $\mathscr{D}^{\prime}$, the dual of $\mathscr{D}$, are the distributions on $W$. In order to define a function of a distribution we first regularize the distribution.

Let $\rho$ be a $C_{c}^{\infty}\left(\boldsymbol{R}^{p}\right)$ function satisfying $0 \leqq \rho \leqq 1, \rho(x)=1$ if $\|x\| \leqq 1$ and $\rho(x)=0$ if $\|x\| \geqq 2$. Set

$$
\gamma_{n}(x)=\rho\left(n_{r} x\right)\left(\int \rho\left(n_{0} t\right) d t\right)^{-1}
$$

where $n_{0}$ is chosen so that

$$
\text { distance }\left(\bar{B}_{n},{\overline{B_{n+1}^{c}}}^{c}\right)>2 / n_{0} \text {. }
$$

Choose $\beta_{n}$ to be a $C_{c}^{\infty}\left(\boldsymbol{R}^{p}\right)$ function satisfying $\beta_{n}=1$ on $B_{n-1}, 0 \leqq$ $\beta_{n} \leqq 1$ and support $\left(\beta_{n}\right) \subset B_{n}$.

If $x$ is in $B_{n}$ and $\gamma_{n}(x-y) \neq 0$ then $\|x-y\| \leqq 2 / n_{0}$ so that $y$ is in $B_{n+1}$. If $\gamma_{n}(x-\cdot)$ denotes the function $y \rightarrow \gamma_{n}(x-y)$ then $\gamma_{n}(x-\cdot)$ is in $C_{c}^{\infty}\left(B_{n+1}\right)$ if $x \in B_{n}$. For any distribution $T$ on $W$ define a function $T_{n}$ by

$$
T_{n}(x)=\beta_{n}(x) T\left(\gamma_{n}(x-\cdot)\right)
$$

for $n$ in $N$. Then $T_{n}$ is in $C_{c}^{\infty}\left(\boldsymbol{R}^{p}\right)$ and support $\left(T_{n}\right) \subset B_{n}$. By the transfer principle $T_{n}$ is in ${ }^{*} C_{c}^{\infty}\left(\boldsymbol{R}^{p}\right)$ and support $\left(T_{n}\right) \subset{ }^{*} B_{n}$ for $n$ in ${ }^{*} N$. Further, since $T_{n} \rightarrow T$ in $\mathscr{D}^{\prime}$ as $n \rightarrow \infty$ in $N$ it follows that

$$
T_{n}(f) \approx T(f)
$$

for all $n$ in ${ }^{*} N-N$ and $f$ in $\mathscr{D}$ where $T_{n}(f)=\left\langle T_{n}, f\right\rangle$. 
The $T_{n}$ are called the regularizations of $T$.

Definition 1. Fix $n$ infinite in ${ }^{*} N$. Let $g$ be a complex valued function of a complex variable defined on the range of $T_{n}$. Then we define $g(T)$ to be $g \circ T_{n}$.

Thus, $g(T)$ is a generalized function in the sense that it is an internal ${ }^{*} \boldsymbol{C}$-valued function defined on ${ }^{*} W$. Since $T_{n}(x)=0$ if $x$ is not in $B_{n}$ we find that $g(T)(x)=g(0)$ if $x$ is not in ${ }^{*} B_{n}$.

Certain generalized functions $h:{ }^{*} W \rightarrow{ }^{*} C$ define distributions $\tau$ according to the rule

$$
\tau(f)=\operatorname{st}(\langle h, f\rangle)
$$

for $f$ in $\mathscr{D}$. Here, st $(\lambda)$ is the standard part of $\lambda$, if $\lambda$ is a finite hypercomplex number, and undefined otherwise. Thus not every function of a distribution will determine a distribution according to (2). If $g$ is a bounded measurable function then $g(T)$ always defines a distribution by (2).

If $T$ is given by a locally $L^{1}$ function $F$ on $W$, (i.e., if $T(f)=$ $\langle F, f\rangle$ for all $f$ in $\mathscr{D}$ ) then $g$ of $T$ is naturally defined within standard distribution theory as the distribution $f \rightarrow\langle g \circ F, f\rangle$ providing the composition is defined. A simple example suffices to show that the distribution determined by $g(T)$ need not be $g \circ F$.

ExAMPLE 2. Let $E$ be the complement of the Cantor set, in $[0,1]$, of measure $1 / 2$ obtained by repeated deletions of $2^{n-1}$ open intervals of length $2^{-2 n}, n=1,2, \cdots$ from $[0,1]$. Let $F$ be the characteristic function of $E$ and define $T(f)=\langle F, f\rangle$. Let $W=$ $(-2,2)$ in $\boldsymbol{R}^{1}$. Choosing $B_{1}$ so that $[0,1] \subset B_{1}$ gives

$$
\begin{aligned}
T_{n}(x) & =T\left(\gamma_{n}(x-\cdot)\right)=\left\langle F, \gamma_{n}(x-\cdot)\right\rangle \\
& =\int F(y) \gamma_{n}(x-y) d y>0 \text { for all } n \text { in } N
\end{aligned}
$$

and all $x$ in $[0,1]$. By the transfer principle $T_{n}(x)>0$ for all $n$ in ${ }^{*} \boldsymbol{N}$ and $x$ in ${ }^{*}[0,1]$. Let $g(x)=1$ if $x \neq 0$ and $g(0)=0$. Then $g(T)(x)=1$ if $x$ is in $*[0,1]$ while $g \circ F(x)=1$ if $x$ is in $E$ and $g \circ F(x)=0$ if $x$ is not in $E$. Choosing $f$ in $C_{c}^{\infty}(-2,2)$ with $f=1$ on $[0,1]$ shows $g(T)(f)=\langle g(T), f\rangle=1$ while

$$
\begin{aligned}
\langle g \circ F, f\rangle & =\int_{W} g(F(x)) f(x) d x \\
& =\int_{E} g(F(x)) f(x) d x \\
& =\int_{E} f(x) d x=\text { measure }(E)=1 / 2 .
\end{aligned}
$$


Thus

$$
g \circ F(f)=\langle g \circ F, f\rangle \neq\langle g(T), f\rangle=g(T)(f) .
$$

The necessity of considering only bounded "smooth" $g$ 's is now demonstrated, if one is to obtain a generalization of composition of functions. In Lemma 5 we obtain such a generalization.

Lemma 3. Let $f$ be in ${ }^{*} L^{1}(K, d x)$ where $K$ is a subset of $\boldsymbol{R}^{p}$. Let $\|f\|_{1} \approx 0$. Then $M=\left\{x\right.$ in $\left.{ }^{*} K: f(x) \neq 0\right\}$ is contained in an internal measurable subset $P$ of ${ }^{*} K$ and measure $(P) \approx 0$. Since

Proof. Let $A_{m}=\left\{x\right.$ in $\left.{ }^{*} K ;|f(x)|>1 / m\right\}$. Then $M=\mathbf{U}_{m \text { in }} A_{m}$.

$$
\|f\|_{1}>\int_{A_{m}}|f| d x>\operatorname{meas}\left(A_{m}\right) / m,
$$

we find that meas $\left(A_{m}\right) \approx 0$ for each $m$ in $N$. By definition $A_{1} \subset A_{2} \subset$ $A_{3} \subset \cdots$.

Extend $\left\{A_{m}\right\}$ to an internal sequence. Let $I=\{m$ in $* N: 2 \leqq$ $k \leqq m$ implies meas $\left(A_{k}\right)<1 / k$ and $\left.A_{k-1} \subset A_{k}\right\} . \quad I$ is internal and contains $N$. Since $N$ is external there is an $\omega$ in $\left({ }^{*} N-N\right) \cap I$. Choose $P=A_{\omega}$. Then meas $(P) \approx 0$ and $\bigcup_{m \text { in } N} A_{m} \subset P$.

Lemma 4. Let $F$ be a locally $L^{1}$ function on $W$. Let $T$ be the distribution $T(f)=\langle F, f\rangle$. For every compact $K$ in $W$ and every infinite $n$ in ${ }^{*} N$

$$
\left\|T_{n}-F\right\|_{1} \approx 0
$$

where \|\|$_{1}$ is the $L^{1}$ norm on ${ }^{*} K$.

Proof. Choose an open set $Q$ with compact closure such that

$$
K \subset Q \subset \bar{Q} \subset W .
$$

For $n$ in $N$ sufficiently large, $\beta_{n}=1$ on $\bar{Q}$, and $x$ in $K$ implies support $\left(\gamma_{n}(x-\cdot)\right) \subset \bar{Q}$. Consequently, for $n$ in $N$ sufficiently large $T_{n}(x)=\left(\gamma_{n} * F\right)(x)$. But $\gamma_{n} * F \rightarrow F$ in $L^{1}(K)$ so for $n$ infinite $\| F-$ $\gamma_{n} * F \|_{1} \approx 0$ while by definition $T_{n}=\gamma_{n} * F$ so $\left\|F-T_{n}\right\|_{1} \approx 0$.

LeMma 5. Let $g$ be a bounded uniformly continuous function defined on a closed interval containing 0 and the range of a locally $L^{1}$ function $F$. Let $T$ be the distribution defined by $F: T(f)=$ $\langle F, f\rangle$ for $f$ in $\mathscr{D}$. Then $g \circ F$ is the distribution determined by $g(T)$. 
Proof. We first must show $g(T)$ is defined. There is a closed interval on which $g$ is defined and which contains the range of $F$. Since $0 \leqq \beta_{n} \leqq 1$ and $\int \gamma_{n} d x=1$ it follows that the range of $\beta_{n}\left(\gamma_{n}^{*} F\right)=$ $T_{n}$ is in the closed interval and so the range of $T_{n}$ is in the domain of $g$ for all $n$ in $N$. By transfer $g\left(T_{n}\right)$ is defined for all $n$ in $* N$.

Fix $n$ infinite and define $g(T)=g\left(T_{n}\right)$.

We are to prove that for every $f$ in $\mathscr{D}$

$$
\langle g(T), f\rangle \approx\langle g \circ F, f\rangle .
$$

Let $K$ be a compact set containing the support of $f$. Let $A$ be an internal subset of ${ }^{*} K$ containing $\left\{x\right.$ in $\left.{ }^{*} K: T_{n}(x) \approx F(x)\right\}$ and having measure $\lambda \approx 0$. Since $g$ is uniformly continuous, $x$ in $* K-$ $A$ implies $g\left(T_{n}(x)\right) \approx g(F(x))$. Let $M$ in $N$ be a bound for $g$. Let $\varepsilon$ be a positive real number. Let $m$ be the Lebesgue measure of $K$. Then

$$
\begin{aligned}
& |\langle g(T), F\rangle-\langle g \circ F, f\rangle| \\
& \quad \leqq \int_{A}\left|g\left(T_{n}\right)-g(F)\right||f| d x+\int_{*_{K-A}}\left|g\left(T_{n}\right)-g(F)\right||f| d x \\
& \quad \leqq 2 M\|f\|_{\infty} \lambda+\varepsilon\|f\|_{\infty} m<2 \varepsilon\|f\|_{\infty} m .
\end{aligned}
$$

Since $\varepsilon$ is arbitrary, this completes the proof.

EXAMPLe 6. Let $\delta$ be the delta function $\delta(f)=f(0), f$ in $\mathscr{D}\left(\boldsymbol{R}^{1}\right)$. Choose $n_{0}=n$ and $B_{n}=(-n, n)$. Then, $\delta_{n}(x)=\gamma_{n}(x)$. For all $n$ in $\boldsymbol{N}$, support $\left(\delta_{n}\right) \subset(-2 / n, 2 / n)$ and $0 \leqq \delta_{n}<\infty$. Thus for $t>0$ in $\boldsymbol{R}$, $0<e^{-t \hat{\delta}_{n}} \leqq 1$ and if $|x|>2 / n$ then $e^{-t \delta_{n}}(x)=1$. By the transfer principle choosing $n$ in ${ }^{*} N-N$ and setting $g(T)=g\left(T_{n}\right)$ we find $0<g(T)<1$ and $g(T)(x)=1$ if $|x|>2 / n$. Thus

$$
\langle g(T), f\rangle \approx\langle 1, f\rangle, f \text { in } \mathscr{D} \text { so }
$$

1 is the distribution determined by $e^{-t}$.

Next we wish to discuss exponentials of distributions in connection with the Trotter product formula. This requires a discussion of the topology of strong resolvent convergence, which we now give. More details on this topology are to be found in the appendix.

DeFinition. Let $S A(K)$ be the set of all selfadjoint operators on a Hilbert space $K$. A neighborhood basis of a selfadjoint operator $S$ in the topology of strong resolvent convergence is given by the collection

$\{G(S, E, \varepsilon): E$ is a finite subset of $K, 0<\varepsilon<\infty\}$ 
where $G(S, E, \varepsilon)=\left\{T\right.$ in $S A(K):\left\|(T+i)^{-1} h-(S+i)^{-1} h\right\|<\varepsilon, h$ in $E\}$. We refer to the elements of ${ }^{*} S A(K)$ as the internal selfadjoint operators on ${ }^{*} K$. Information about ${ }^{*} S A(K)$ may be obtained by transfer. For example, "For all $T$ in ${ }^{*} S A(K), \lambda$ in ${ }^{*} C, \lambda \neq 0, \|(T+$ $\lambda)^{-1}|| \leqq|\operatorname{Im}(\lambda)|^{-1}$ " is obtained by transfer from "For all $S$ in $S A(K), \lambda$ in $C, \lambda \neq 0,\left\|(S+\lambda)^{-1}\right\| \leqq|\operatorname{Im}(\lambda)|^{-1}$.

LEMMA 7. An internal selfadjoint operator $T$ is near standard in the topology of generalized strong convergence if and only if there is an $S$ in $S A(K)$ such that

$$
\left\|(T+i)^{-1} h-(S+i)^{-1} h\right\| \approx 0 \text { for all } h \text { in } K .
$$

Proof. $S=\operatorname{st}(T)$ iff $T$ is in

$$
\{\cap * G(S, E, r): E \text { is finite, } 0<r<\infty\} .
$$

But $T$ is in ${ }^{*} G(S, E, r)$ iff

$$
\left\|(T+i)^{-1} h-(S+i)^{-1} h\right\|<r \text { for all } h \text { in } E \text {, since } E \text { is finite. }
$$

Lemma 8. Let $S$ belong to $S A(K)$ and $T$ to ${ }^{*} S A(K)$. Then $T \approx S$ if and only if

$$
e^{i t T} h \approx e^{i t S h}
$$

for all finite $t$ in ${ }^{*} \boldsymbol{R}$ and $h$ in $K$.

If $T$ and $S$ are bounded from below by some finite number. Then (3) may be replaced with

$$
e^{-t T} h \approx e^{-t S}
$$

for all finite nonnegative $t$ in ${ }^{*} \boldsymbol{R}$ and $h$ in $K$.

Proof. We give the proof that $T \simeq S$ implies (3) for $t$ positive and finite. For any $P$ in ${ }^{*} S A(K)$ let $R(P)=(-i T+1)^{-1}$ and $E(P)=e^{i P}$. Let $h$ be an arbitrary element of $K$. Then, by definition of $T \approx S$, one has $R(T) h \approx R(S) h$. Since $R(T), R(S)$ are contractions it follows that $R(T) a \approx R(S) b$ for all near standard $a, b$ in ${ }^{*} K$ with $a \approx b$. In particular, since $s \rightarrow E(s S)$ is strongly continuous we conclude that $E(s S) h$ is near standard for all finite $s$ in $* R$, so that $\|(R(T)-R(S)) E(s S) h\| \approx 0$. Consequently, for all positive real $\lambda,\|E((t-s) T)(R(T)-R(S)) E(s S) h\|<\lambda$. Transferring equation (2.27) of Reference 3 , page 501, gives

$$
R(T)(E(t T)-E(t S)) R(S)=i \int_{0}^{t} E((t-s) T)(R(T)-R(S)) E(s S) d s
$$


and provides the estimate $\|R(T)(E(t T)-E(t S)) R(S) h\| \leqq \lambda t$ for all positive real $\lambda$ so that $R(T) E(t T) h \approx E(t S) R(S) h$. For all $t$ in ${ }^{*} \boldsymbol{R}$, $E(t S) R(S)=R(S) E(t S)$, follows by transferring the corresponding statement with $t$ in $\boldsymbol{R}$. Since $E(t S) h$ is near standard we conclude

$$
R(T) E(t T) h \approx R(T) E(t S) h .
$$

Next we argue

$$
\begin{aligned}
E(t T) R(S) h & \approx E(t T) R(T) h=R(T) E(t T) h \approx R(T) E(t S) h \\
& \approx R(S) E(t S) h=E(t S) R(S) h .
\end{aligned}
$$

Since the range of $R(S)$ is dense in $K$ and since $E(t S), E(t T)$ have finite norms we conclude that $E(t T) h \approx E(t S) h$.

If $t$ is finite and negative the proof follows as above upon replacing $T$ and $S$ by $-T$ and $-S$ respectively.

For the proof of the converse we assume $e^{i t T} h e^{i t S} h$ for all finite $t$ in ${ }^{*} \boldsymbol{R}$ and $h$ in $K$. From Lemma 12, appendix, it suffices to prove

$$
(-i T+1)^{-1} h \approx(-i S+1)^{-1} h
$$

for all $h$ in $K$. This is done via the transferred Laplace transform formula $(-i T+1)^{-1} h=\int_{0}^{\infty} e^{-t} e^{i t T} h d t$. Let $\lambda, \in>0$ be arbitrary real numbers. First choose a positive real number $c$ so that $\int_{0}^{\infty} e^{-t} d t<\varepsilon$.

$$
\begin{gathered}
\left\|(-i T+1)^{-1} h-(-i S+1)^{-1} h\right\| \leqq 2 \varepsilon+\int_{0}^{c} e^{-t}\left\|\left(e^{i t T}-e^{i t S}\right) h\right\| d t \\
\leqq 2 \varepsilon+\int_{0}^{c} e^{-t} \lambda d t<2 \varepsilon+\lambda .
\end{gathered}
$$

Thus, $(-i T+1)^{-1} h \approx(-i S+1)^{-1} h$ for all $h$ in $K$.

The proof for the case of $T$ and $S$ finitely bounded below follows similarly.

REMARK. The proof above closely parallels Kato's proof [3, p. 502] of an analogous standard result.

REMARK. The restriction of (3) to finite $t$ in ${ }^{*} \boldsymbol{R}$ is not superfluous. Let $n$ be a positive infinite integer, $T=1 / n, S=0$, and $t=n$. Then $T \approx S$ but

$$
e^{i t T} h=e^{i} h \not h=e^{i t S} h .
$$

EXAMPLe 8. Let $h_{0}: \boldsymbol{R}^{p} \rightarrow[0, \infty)$ be continuous and suppose $e^{-t h_{0}}$ is in $L^{1}$ and is positive definite. Let $H_{0}=F M\left(h_{0}\right) F^{-1}$ where $F$ is the unitary Fourier transform on $L^{2}\left(\boldsymbol{R}^{p}\right)$ and $M\left(h_{0}\right)$ is the selfadjoint 
operator given as multiplication by the function $h_{0}$. Examples of such operators are given by $h_{0}(k)=|k|^{2}$ when $H_{0}=-\Delta$ and $h_{0}(k)=$ $\sqrt{|k|^{2}+m^{2}}, m>0$. For a complete discussion of such operators, see [2]. Let $\tau$ be a real distribution on $\boldsymbol{R}^{p}$ which satisfies for some $0<a<1$ and $b>0$

$$
\pm \tau\left(|f|^{2}\right) \leqq a\left(H_{0} f, f\right)+b\|f\|^{2}
$$

for all $f$ in $C_{c}^{\infty}\left(\boldsymbol{R}^{p}\right)$. Such a $\tau$ is called a small form perturbation of $H_{0}$. As shown in [2] the form sum of $H_{0}$ and the continuous extension of $\tau$ to the form domain of $H_{0}$ is selfadjoint and bounded below. We denote this standard operator by $H_{0}+\tau$. As concrete examples choose $H_{0}=-\Delta$ and $\tau$ the delta function concentrated on the surface of a sphere in $p>1$ dimensions and $\tau=\delta$, the delta function in $p=1$ dimensions. Also when $p=1, \tau=e^{x} \cos \left(e^{x}\right)$ is a small form perturbation of $-\Delta$. See [2] for more examples.

Let $\tau_{n}$ denote the regularizations of $\tau$. Then, defining $H_{0}+\tau_{n}$ as the operator sum, it follows from the strong convergence of the resolvents of $H_{0}+\tau_{n}$ to those of $H_{0}+\tau$, [2], that

$$
H_{0}+\tau_{n} \approx H_{0}+\tau
$$

for all positive infinite integers, $n$.

EXAMPLE 9. Let $H_{0}, V$ be nonnegative selfadjoint operators on a Hilbert space $K$. Suppose $D\left(\left(H_{0}\right)^{1 / 2}\right) \cap D\left((V)^{1 / 2}\right)$ is dense. Define the truncations of $V$ by

$$
V_{n}=\left\{\begin{array}{lll}
V & \text { if } & V \leqq n \\
0 & \text { if } & V>n
\end{array}\right.
$$

via the spectral theorem. Let $H_{0}+V$ denote the standard selfadjoint operator defined as the form sum of $H_{0}$ and $V$ in [1]. Then, since $\left(H_{0}+V_{n}+i\right)^{-1}$ converges strongly to $\left(H_{0}+V+i\right)^{-1}$, [1], we conclude

$$
H_{0}+V \approx H_{0}+V_{n}
$$

for all positive infinite integers, $n$. See [1] and [4] for concrete examples.

We now proceed to discuss the Trotter product formula for given form sums. A discussion of form sums may be found in [1]. Here $H_{0}$ is a selfadjoint operator on a Hilbert space $K$ and $H$ is the selfadjoint operator on $K$ given as the form sum of $H_{0}$ and $V$ in the following three cases:

Case 1. $V$ is a selfadjoint operator and the operator sum $H_{0}+$ 
$V$ is essentially selfadjoint.

Case 2. $H_{0}$ and $V$ are bounded below selfadjoint operators with dense form domain intersection; and

Case 3. $V$ is a small Hermitian form perturbation of $H_{0}$.

In Case $1, H$ is just the closure of $H_{0}+V$ and the Trotter product formula holds [12]:

$$
e^{\lambda t I I}=\underset{n \rightarrow \infty}{\operatorname{strong}} \operatorname{limit}\left(e^{\lambda t I I_{0} / n} e^{\lambda t V / n}\right)^{n}
$$

uniformly for $t$ in compact subsets of $[0, \infty)$, where $\lambda= \pm i$. If $H_{0}, V$ are bounded below then (6) also holds with $\lambda=-1$.

In Case 2 Kato has shown [4] that (6) holds for $\lambda=-1$ but in general (6) is not known for $\lambda= \pm i$ and in fact there has been essentially no progress in extending (6) for $\lambda= \pm i$ beyond Case 1.

In Case 3, not only is Trotter's product formula unknown for $\lambda= \pm i$ and $\lambda=-1$ but in general it makes no sense. For example, if $H_{0}=-d^{2} / d x^{2}$ and $V=\delta$, the Dirac delta function on $L^{2}\left(\boldsymbol{R}^{1}\right)$, then $e^{\lambda V / n}$ is undefined in any standard sense. In some examples of Case 3 such as $H_{0}=-d^{2} / d x^{2}$ and $V(x)=e^{x} \cos \left(e^{x}\right)$ the formula (6) makes sense but its validity is unknown for $\lambda=-1$.

Elementary nonstandard analysis provides a convenient framework for obtaining a type of product formula in the cases discussed above.

Theorem 10. A Product Formula:

Let $H_{0}, H$ be in $S A(K)$ and $V$ in ${ }^{*} S A(K)$ where $K$ is a separable Hilbert space. Suppose the closure $\left[H_{0}+V\right]$ of the operator sum of $H_{0}$ and $V$ is in ${ }^{*} S A(K)$. Also assume $H \approx\left[H_{0}+V\right]$.

Then there is an $N$ in ${ }^{*} N$ such that for all $n>N, h$ in $K$ and nonnegative finite $t$ in ${ }^{*} \boldsymbol{R}$ :

$$
e^{\lambda t H} h \approx\left(e^{\lambda t H_{0} / n} e^{\lambda t V / n}\right)^{n} h
$$

where $\lambda= \pm i$.

If also, $H, H_{0}, V>c$ for some $c$ in $R$ then (7) holds with $\lambda=-1$.

Proof. By Lemma 8

$$
e^{\lambda t H} h \approx e^{\lambda t\left[H_{0}+V\right]} h
$$

for all $h$ in $K$ and all finite $t$ in ${ }^{*} \boldsymbol{R}$.

Fix $\alpha$ to be a positive infinitesimal. Fix $h$ in $K$ and $M$ in $N$. 
It follows from the transferred Trotter product formula of Case 1 that there is a $Q(h, M)$ in ${ }^{*} N$, (we suppress the $\alpha$ dependence), such that $q>Q(h, M)$ implies

$$
\left\|e^{\lambda t\left[M_{0}+V\right]} h-\left(e^{\lambda t H_{0} / q} e^{\lambda t V / q}\right)^{q} h\right\|<\alpha
$$

for all $t$ in *[0,M]. Let $Q(h)$ be an upper bound for $\{Q(h, M): M$ is in $N$, which exists by [8, page 59]. Then $q>Q(h)$ implies

$$
e^{\lambda t I I} h \approx\left(e^{\lambda t H_{0} / q} e^{\lambda t V / q}\right)^{q} h
$$

for all finite $t$ in $*[0, \infty]$.

Let $C$ be a countable basis for $K$. Let $Q$ be an upper bound for $\{Q(h): h$ is in $C\}$, which again exists by [8]. Let $q>Q, t$ in $*[0, \infty)$ be finite and $h$ in $K$ be arbitrary. Given $\beta$ in $(0, \infty)$ choose $k$ in $C$ so that $\|h-k\|<\beta$. Then

$$
\begin{aligned}
\| e^{\lambda t H} h & -\left(e^{\lambda t H_{0} / q} e^{\lambda t V / q}\right)^{q} h \| \\
\leqq & \left\|\left(e^{\lambda t H}(h-k)\right)\right\| \\
& +\left\|\left(e^{\lambda t H}-\left(e^{\lambda t H_{0} / q} e^{\lambda t V / q}\right)^{q}\right) k\right\| \\
& +\left\|\left(e^{\lambda t H_{0} / q} e^{\lambda t V / q}\right)^{q}(k-h)\right\|<3 \beta,
\end{aligned}
$$

since all operators appearing are contractions and the 2nd term being infinitesimal is infact less than $\beta$. As $\beta$ is arbitrary in $(0, \infty)$ the difference in (11) is infinitesimal and the proof is complete.

REMARKs. (a) The theorem applies to Example (8) with $\lambda= \pm i$ and in case the distribution is nonnegative, such as with the delta functions, $\lambda$ may also be taken as -1 . The theorem also applies to Example (9) with $\lambda= \pm i,-1 . H$ is the form sum and in these examples, the $V$ is either a truncation or a regularization with positive infinite integral index and so is a bounded element of ${ }^{*} S A(K)$. Consequently $\left[H_{n}+V\right]=H_{0}+V$.

(b) In case $\lambda=-1$ it suffices to assume $H_{0}, V>C$ as we will see in the appendix, Example 6.

(c) If $V$ is in $S A(K)$ rather than ${ }^{*} S A(K)$ then the formula (7) is equivalent to the standard formula (6).

(d) In [9] we used a preliminary form of this product formula to express the dynamics of a singularly perturbed quantum system in terms of a nonstandard Feynman path integral.

(e) If $K$ is not separable, one still obtains a product formula but then the $N$ in ${ }^{*} N$ depends on the $h$ in $K$.

EXAmple 11. In Example 6 we observed that $e^{-t \delta}$ could be identified with 1 . However, this identification may not be made in 
the present context, for we have just seen that

$$
e^{-t\left(-d^{2} / d x^{2}+\delta\right)} h \approx\left(e^{t\left(d^{2} / d x^{2} / n\right)} e^{-t \delta / n}\right)^{n} h
$$

or all $n$ sufficiently large. Had we also identified $e^{-t \bar{s} / n}$ with 1 we would have obtained

$$
e^{-t\left(-d^{2} / d x^{2}+\delta\right)} h \approx e^{t\left(d^{2} / d x^{2}\right)} h
$$

which implies $-d^{2} / d x^{2}+\delta=d^{2} / d x^{2}$ and this is false.

APPEndix. The Strong Resolvent Topology.

In this appendix $T$ will denote an element of ${ }^{*} S A(K)$ and $S$ an element of $S A(K)$, for $K$ a complex Hilbert space. Conditions for and consequences of $T \approx S$ will be investigated. There are closely related standard results which are obtained by considering a sequence, $\left\{T_{n}\right\}$, in $S A(K)$ and its (possible) limit $S$, in the strong resolvent topology. One could obtain corresponding nonstandard results by transferring to $T=T_{n}, n$ infinite and such a procedure would be sufficient for the examples considered in this paper: $T_{n}=$ $H_{0}+V_{n}$. However, in this appendix we are not assuming that $T$ in ${ }^{*} S A(K)$ has this special form and so, in general simple transfer arguments are not necessarily sufficient to establish the desired results.

Definition. The finite resolvent set of $T$, denoted $\rho(T)$, is the set of all finite $\lambda$ in ${ }^{*} C$ such that $T-\lambda$ is $1-1$, onto and $\|(T-$ $\lambda)^{-1} \|$ is finite.

ExAMPLES. By transfer of the resolvent equation
(a) $\left\{\right.$ finite $\left.\lambda \varepsilon^{*} C: \operatorname{Im}(\lambda) \approx 0\right\} \subset \rho(T)$;
(b) If $T \geqq 0$ then

$$
\text { \{inite } \left.\lambda \varepsilon^{*} R: \lambda<0, \lambda \approx 0\right\} \subset \rho(T) \text {. }
$$

Lemma 12. Let $\rho$ denote the intersection of the finite resolvent sets of $S$ and $T$. Then

(i) $T \approx S$ if there is a $\lambda$ in $\rho$ so that

$$
\left\|(T-\lambda)^{-1} h-(S-\lambda)^{-1} h\right\| \approx 0
$$

for all $h$ in $K$.

(ii) If $T \approx S$ then (12) holds for all $\lambda$ in $\rho$.

Proof. The proof follows from a resolvent equation as in [3, page 429] together with the fact that operators with finite norm take infinitesimal vectors into infinitesimal vectors. 
Definition. Let $D$ be a subspace of the domain of $S, D(S)$. $D$ is core for $S$ if $S$ restricted to $D$ has exactly one selfadjoint extension.

Lemma 13. Let $D$ be a core for $S$. If $D \subset D(T)$ and $\| S f-$ $T f \| \approx 0$ for all $f$ in $D$ then $T \approx S$.

Proof. $E=(S+i)(D)$ is dense in $K$. Let $g$ in $K$ be arbitrary and let $\delta$ be a positive real number. Choose $f$ in $E$ so that $\| f$ $g \|<\delta / 3$. Then

$$
\begin{aligned}
\|(T+ & i)^{-1} g-(S+i)^{-1} g \| \\
\leqq & \left\|(T+i)^{-1}(g-f)\right\|+\left\|\left[(T+i)^{-1}-(S+i)^{-1}\right] f\right\| \\
& +\left\|(S+i)^{-1}(f-g)\right\| \\
\leqq & \|g-f\|+\left\|(T+i)^{-1}(T-S)(S+i)^{-1} f\right\|+\|f-g\| \\
\leqq & 2 \delta / 3+\left\|(T-S)(S+i)^{-1} f\right\|<\delta .
\end{aligned}
$$

REMARK. See [3, page 424] for analogous standard result.

EXAMPLE 14. Let $H_{0}, V$ be in $S A(K)$. Let $V_{n}$ be the truncations of $V$ as in Example 9. If the operator sum $H_{0}+V$ is essentially selfadjoint then $H_{0}+V_{n} \approx\left[H_{0}+V\right]$, the closure of $H_{0}+V$, for all positive infinite $n$. This follows directly from Lemma 13 because if $f \in D(V)$ then $V f \approx V_{n} f, n \in{ }^{*} N-N$, by dominated convergence. Thus,

$$
\left(H_{0}+V_{n}\right) f \approx\left[H_{0}+V\right] f
$$

for all $f$ in the core $D\left(H_{0}+V\right)$ for $\left[H_{0}+V\right]$.

REMARK. It may happen that $T \approx S$ but that there is no core $D$ for $S$ on which $T$ and $S$ nearly agree. For example let $V$ be a nonnegative locally $L^{1}$ function on $\boldsymbol{R}^{1}$ which is not $L^{2}$ on any interval. Let $V_{n}$ be the truncation of $V: V_{n}(x)=V(x)$ if $V(x) \leqq n$ and $V_{n}(x)=0$ otherwise. Let $S=-d^{2} / d x^{2}$ and $T=$ the form sum of $S$ and $V_{n}, n$ infinite. If $f$ is in $D(T)=D(S)$ and in $K$ then $f$ is continuous and unless $f$ is identically zero, $|f|$ is bounded away from zero on some interval. Consequently $\left\|V_{n} f\right\|$ is infinite. Since $\|S f\|$ is finite it follows that

$$
f \in K \cap D(T) \longrightarrow\|T f\| \text { infinite. }
$$

Thus, there is no converse to the previous lemma. On the other hand we do have

Lemma 15. If $T \approx S$ then to each $h$ in $D(S)$ there is an $h^{\prime}$ in 
$D(T)$ with $h^{\prime} \approx h$ and $T\left(h^{\prime}\right) \approx S(h)$.

Proof. Define $h^{\prime}=(T+i)^{-1}(S+i) h$. Then $h^{\prime} \in D(T)$ and $h^{\prime}=$ $h+\left((T+i)^{-1}-(S+i)^{-1}\right)(S+i) h \approx h$. Further, $(T+i) h^{\prime}=(S+i) h$ so that $T h^{\prime} \approx S h$.

EXAMPLE 16. Let $c$ belong to $R$. Let $H_{0} \in S A(K), V \in{ }^{*} S A(K)$ be * bounded and $H_{0}, V \geqq c$. Suppose $H \in S A(K)$ and $H \approx H_{0}+V$. By Lemma 15, for each $h$ in $D(H)$ there is an $h^{\prime}$ in $D\left(H_{0}+V\right)$ such that $H h \approx\left(H_{0}+V\right) h^{\prime}$ and $h \approx h^{\prime}$. Thus

$$
\begin{aligned}
(H h, h) & \approx\left(H h, h^{\prime}\right) \approx\left(\left(H_{0}+V\right) h^{\prime}, h^{\prime}\right) \\
& \geqq c\left\|h^{\prime}\right\|^{2} \\
& \approx c\|h\|^{2} .
\end{aligned}
$$

Since $(H h, h)$ and $c\|h\|^{2}$ are in $\boldsymbol{R}$ we conclude $H \geqq c$.

For bounded operators there are no domain problems so we expect

LEMMA 17. Let $S$ be bounded and let $T$ be everywhere defined with finite norm.

(a) If $T h \approx S h$ for all $h$ in a dense subspace $D$ of $K$, then $T \approx S$.

(b) If $T \approx S$ then $T h \approx S h$ for all $h$ in $K$.

Proof. Since $D$ is dense and $S$ is bounded, $D$ is a core for $S$ so part (a) follows from Lemma 13.

For part (b), assume $T \approx S$ so that $(T+i)^{-1} h \approx(S+i)^{-1} h$ for all $h$ in $K$. Since $T$ has finite norm $h \approx(T+i)(S+i)^{-1} h$ for all $h$ in $K$. Let $g=(S+i)^{-1} h$. Then $(S+i) g \approx(T+i) g$ for all $g$ in (the range of $\left.(S+i)^{-1}\right)=K$.

One of the reasons for the utility of the topology of strong resolvent convergence in perturbation theory is

LEMMA 18. Let $\phi$ be a bounded Borel function on the real line which is continuous except on a closed set of $S$ spectral measure zero. If $T \approx S$ then $\phi(T) h \approx \phi(S) h$, for all $h$ in $K$.

Proof. With only minor modifications, (replacing certain "="'s with “ $\approx$ "'s), the proof of Faris, [1, pages 40-42] for an analogous standard result works here.

Lemma 19. Let $T$ be near standard in the strong resolvent 
topology. Then for all $h$ in $K$ and all $\lambda \approx 0$

$$
e^{i \lambda T} h \approx h \text {. }
$$

If also $T \geqq c, c$ in $\boldsymbol{R}$, then

$$
e^{-\lambda T} h \approx h .
$$

Proof. By Lemma 8

$$
e^{i \lambda T} h \approx e^{i \lambda S} h \approx h
$$

by strong continuity of $t \rightarrow e^{i t S}$.

EXAMPLE 20. Let $T=n, n$ a positive, infinite integer. Let $\lambda=1 / n \approx 0$. Then $e^{i \lambda T} h=e^{i} h \approx h$ so $T$ is not near standard even though $e^{i t T} h$ is near standard for all $t$ in $* \boldsymbol{R}$.

Similarly $(1+i \lambda T)^{-1} h \approx \hbar$ while from Lemma 19 and the Laplace transform formula

$$
(1+i \lambda G)^{-1}=\int_{0}^{\infty} e^{-t} e^{-\imath \lambda t G} h d t
$$

it follows that $(1+i \lambda G)^{-1} h \approx h$ for all near standard $G$. Thus, $(T+c)^{-1} h$ near standard for all $h$ in $K$ does not imply that $T$ is near standard in ${ }^{*} S A(K)$. We next discuss when $(T+c)^{-1} h$ near standard does imply $T$ is near standard. We shall use the following hybrid notation.

For $a_{n}$ in ${ }^{*} \boldsymbol{R}$ we let " $a_{n} \rightarrow 0, n$ in $\boldsymbol{N}$ " indicate that for every real $0<\varepsilon<\infty$ there is an $N$ in $N$ such that $n>N$ implies $\left|a_{n}\right|<\varepsilon$.

Lemma 21. If $h_{n}$ is near standard in ${ }^{*} K$, if $h$ is in ${ }^{*} K$ and if

$$
\left\|h_{n}-h\right\| \longrightarrow 0, \quad n \text { in } N
$$

then $h$ is near standard.

Proof. Though this lemma is a special case of [10, 8.4.29]. We include the following elementary proof:

Let $h_{n}=k_{n}+\varepsilon_{n}, k_{n}$ in $K, \varepsilon_{n} \approx 0$. Then $\left\|k_{n}-h\right\| \leqq\left\|k_{n}-h_{n}\right\|+$ $\left\|h_{n}-h\right\| \rightarrow 0, n$ in $N$, so that $\left\|k_{n}-k_{m}\right\|<\left\|k_{n}-h\right\|+\left\|h-k_{m} \rightarrow 0\right\|$ as $n, m \rightarrow \infty$ in $N$. Thus $\left\{k_{n}\right\}$ is a Cauchy sequence in $K$. Let $k=$ $\lim _{n \rightarrow \infty} k_{n}$. Then $k$ is in $K$ and for every $\delta$ in $(0, \infty)\|h-k\| \leqq$ $\left\|h-h_{n}\right\|+\left\|h_{n}-k_{n}\right\|+\left\|k_{n}-k\right\|<\delta$, by choosing $n$ sufficiently large. Thus, $h \approx k$.

LEMMA 22. Suppose that for some finite $\lambda$ in ${ }^{*} \boldsymbol{C}$ with $\operatorname{Im}(\lambda) \approx$ $0,(T-\lambda)^{-1} h$ is near standard for all $h$ in $K$. Then, for all finite 
$\rho$ in ${ }^{*} \boldsymbol{C}$ with $\operatorname{Im}(\rho) \nRightarrow 0$ and $\operatorname{sign}(\operatorname{Im}(\lambda))=\operatorname{sign}(\operatorname{Im}(\rho))$ and for all $h$ in $K,(T-\rho)^{-1} h$ is near standard.

Proof. It suffices to consider those $\rho^{\prime}$ s satisfying $|\rho-\lambda|<$ $|\operatorname{Im}(\lambda)|$ and $|\rho-\lambda| \approx|\operatorname{Im}(\lambda)|$. For such $\rho$ 's there is the formula $(T-\rho)^{-1}=\sum_{j=0}^{\infty}(\rho-\lambda)^{k}(T-\lambda)^{-(k+1)}$. The lemma then follows from the observation that since $(T-\lambda)^{-1}$ maps standard vectors into near standard vectors and since $\left\|(T-\lambda)^{-1}\right\|$ is finite, $(T-\lambda)^{-1}$ in fact maps near standard vectors into near standard vectors so that $(T-\lambda)^{-(k+1)}$ maps standard vectors into near standard ones.

REMARK. See Kato [3, page 427] for an analogous standard result.

THEOREM 23. In order that $T$ be near standard it is necessary and sufficient that both

(a) for some finite, positive and noninfinitesimal $c,(T \pm i c)^{-1} h$ be near standard for all $h$ in $K$; and

(b) $(1+i \in T)^{-1} h \approx h$

for all positive infinitesimal $\varepsilon$ and all $h$ in $K$, be true.

Proof. The necessity follows from Lemma 12 and the discussion in Example 20. For sufficiency, define, by Lemma 22,

$$
R( \pm i a) h=\operatorname{st}\left((T \pm i a)^{-1} h\right)
$$

for all positive, finite noninfinitesimal $a$ and all $h$ in $K$.

Then

$$
\begin{aligned}
& (R(x)-R(y)) h \\
& \quad \approx\left((T+x)^{-1}-(T+y)^{-1}\right) h \\
& \quad=(y-x)(T+x)^{-1}(T+y)^{-1} h .
\end{aligned}
$$

But

$$
\begin{aligned}
& \left\|(T+x)^{-1}(T+y)^{-1} h-R(x) R(y) h\right\| \\
& \leqq\left\|(T+x)^{-1}\left((T+y)^{-1}-R(y)\right) h\right\| \\
& \quad+\left\|\left((T+x)^{-1}-R(x)\right) R(y) h\right\| \approx 0
\end{aligned}
$$

because $\left\|(T+x)^{-1}\right\|$ has finite norm. Thus $(R(x)-R(y)) h \approx(y-$ $x) R(x) R(y) h$ and since both sides are standard the pseudo-resolvent equation results:

$$
R(x)-R(y)=(y-x) R(x) R(y) .
$$

The proof may now be completed as in Kato's proof [3, page 503] of an analogous standard result. 
THEOREM 24. In order that $T$ be near standard it is necessary and sufficient that both

$$
e^{\lambda s T} h \approx h
$$

for all positive infinitesimal s; and

$$
e^{\lambda t T} h
$$

is near standard for all finite $t \geqq 0$ in ${ }^{*} \boldsymbol{R}$

be true for all $h$ in $K$ with both $\lambda=+i$ and $\lambda=-i$.

If $T \geqq c$ for some $c$ in $\boldsymbol{R}$ and if (13) and (14) both hold with $\lambda=-1$ then $T$ is near standard.

Proof. We give the proof in case $\lambda= \pm i$. The case of $\lambda=-1$ follows similarly. The necessity follows from Lemmas 8 and 19 .

For the proof of sufficiency, fix $h$ in $K$, finite $c>0$ in ${ }^{*} \boldsymbol{R}$, $c \approx 0$. The Riemann integrals on $*[0, \infty)$

$$
\int_{0}^{\infty} e^{-c t} e^{ \pm i t T} h d t
$$

are defined by the strong continuity of the integrand.

Fix $\varepsilon>0$ in $\boldsymbol{R}$. Choose $b>0$ in $\boldsymbol{R}$ so that

$$
\int_{b}^{\infty} e^{-c t} d t<\varepsilon / 2\|h\| .
$$

Let $I$ be the collection of $z$ 's in ${ }^{*}[0, \infty)$ which satisfy

$$
\begin{gathered}
t, s \text { in }[0, b],|t-s|<z \\
\text { imply } \\
\left\|\left(e^{i t T}-e^{i s T}\right) h\right\|<\varepsilon c /\left(2\left(1-e^{-c b}\right)\right) .
\end{gathered}
$$

$I$ is internal. The semigroup property and (13) show that $I$ contains all positive infinitesimals. Consequently, there is a $\delta$ in $I \cap$ $(0, \infty)$.

Let $a_{k}=k \delta$ for $k=0,1, \cdots, n$, where $n$ in $N$ is chosen so that $n \delta<b$ while $(n+1) \delta \geqq b$. Set $a_{n+1}=b$. Let

$$
h_{\varepsilon}=\sum_{k=0}^{n-1} e^{i k \delta T} h \int_{a_{k}}^{a_{k+1}} e^{-c t} d t .
$$

$h_{\varepsilon}$ is near standard being a finite sum of near standard vectors.

$$
\begin{aligned}
& \left\|\int_{0}^{\infty} e^{-c t} e^{i t T} h d t-h_{\varepsilon}\right\| \\
& \quad \leqq \sum_{k=0}^{n-1} \int_{a_{k}}^{a_{k+1}} e^{-c t}\left\|\left(e^{i t T}-e^{i k \hat{o} T}\right) h\right\| d t
\end{aligned}
$$




$$
\begin{aligned}
& +\int_{b}^{\infty} e^{-c t}\left\|e^{i t T} h\right\| d t \\
\leqq & \int_{0}^{b}\left(e^{-c t} \varepsilon c / 2\left(1-e^{-c b}\right)\right) d t+\frac{\varepsilon}{2} \\
= & \varepsilon .
\end{aligned}
$$

By Lemma 21,

$$
\int_{0}^{\infty} e^{-\varepsilon t} e^{ \pm i t r} h d t
$$

is near standard. From the Laplace transform formulas

$$
(T \pm i c)^{-1}=\mp i \int_{0}^{\infty} e^{-c t} e^{ \pm i t T} d t
$$

we conclude that $(T \pm i c)^{-1} h$ is near standard. We have thus verified (a) of Theorem 23. We next verify (b).

Let $\varepsilon>0, \varepsilon \approx 0$. Choose $\beta>0$ to be infinite in such a way that $\in \beta \approx 0$. Then

$$
\begin{aligned}
\|(1+ & i \varepsilon T)^{-1} h-h \| \\
= & \left\|\int_{0}^{\infty} e^{-t} e^{+i \varepsilon t T} h d t-h\right\| \\
\leqq & \int_{0}^{\beta} e^{-t}\left\|e^{i \varepsilon t T} h-h\right\| d t \\
& +2\|h\| \int_{\beta}^{\infty} e^{-t} d t \\
\approx & 0
\end{aligned}
$$

since $0<t<\beta \rightarrow \varepsilon t \approx 0 \rightarrow e^{i \varepsilon t T} h \approx h . \quad$ Thus $(1+i \varepsilon T)^{-1} h \approx h$ and this theorem follows from Theorem 23.

See Example 20 for a remote $T$. Notice that (13) fails, in other words, the semigroup is not strongly S-continuous.

\section{REFERENCES}

1. W. Faris, Self-Adjoint Operators, Springer-Verlag, (1975).

2. I. Herbst and A. Sloan, Perturbation of translation invariant positivity preserving semigroups on $L^{2}\left(R^{N}\right)$, Trans. Amer. Math. Soc., 236 (1978), 325-360.

3. T. Kato, Perturbation Theory for Linear Operators, Springer-Verlag, (1966).

4. - Trotter's product formula for an arbitrary pair of selfadjoint contraction semigroups, Berkeley preprint.

5. D. Laugwitz, Eine einfuhrung der $\delta$-funktionen, S.-B. Bayerische Akad. Wiss., 4 (1958), 41-59.

6. W. Luxemburg, Nonstandard Analysis. Lectures on A. Robinson's Theory of Infinitesimal and Infinitely Large Numbers, Mimeographed Notes, California Institute of Technology, (1962). 
7. A. Robinson, Nonstandard Analysis, North-Holland, (1974).

8. A. Robinson and A. Lightstone, Non-Archimedian Fields and Asymptotic Expansions, North-Holland, (1975).

9. A. Sloan, An application of the nonstandard Trotter product formula, J. Math. Phys., 18 (1977), 2495-2496.

10. K. Stroyan and W. Luxemburg, Introduction to the Theory of Infinitesimals, Academic Press, (1976).

11. J. Thurber and J. Katz, Applications of Fractional Powers of Delta Functions, in "Victoria Symposium on Nonstandard Analysis", A. Hurd and P. Loeb, eds., Springer-Verlag, (1974).

12. H. Trotter, Approximation of semigroups of operators, Pacific J. Math., 8 (1958), 887-919.

Received August 4, 1977 and in revised form January 11, 1978. Supported in part by NSF MCS $76-07543$.

Georgia Institute of Technology

Atlanta, GA 30332 


\section{PACIFIC JOURNAL OF MATHEMATICS}

EDITORS

RICHARD ARENS (Managing Editor)

University of California

Los Angeles, California 90024

C. W. Curtis

University of Oregon

Eugene, OR 97403

C. C. MOORE

University of California

Berkeley, CA 94720
J. DUGUNDJI

Department of Mathematics University of Southern Californı Los Angeles, California 90007

R. Finn and J. Milgram Stanford University Stanford, California 94305

\section{ASSOCIATE EDITORS}

E. F. BECKENBACH

B. H. NEUMANN

F. WOLF

K. YoSHIDA

\section{SUPPORTING INSTITUTIONS}

UNIVERSITY OF BRITISH COLUMBIA
CALIFORNIA INSTITUTE OF TECHNOLOGY
UNIVERSITY OF CALIFORNIA
MONTANA STATE UNIVERSITY
UNIVERSITY OF NEVADA, RENO
NEW MEXICO STATE UNIVERSITY
OREGON STATE UNIVERSITY
UNIVERSITY OF OREGON

UNIVERSITY OF SOUTHERNEALIFORNIA STANFORD UNIVERSITY UNIVERSITY OF HAWAII UNIVERSITY OF TOKYO UNIVERSITY OF UTAH WASHINGTON STATE UNIVERSITY UNIVERSITY OF WASHINGTON 


\section{Pacific Journal of Mathematics \\ Vol. 79 , No. 1 \\ May, 1978}

Teófilo Abuabara, A remark on infinitely nuclearly differentiable

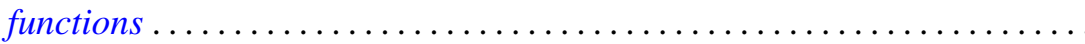

David Fenimore Anderson, Projective modules over subrings of $k[X, Y]$

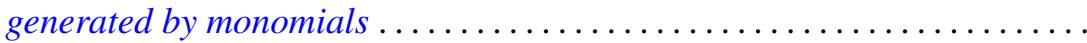

Joseph Barback and Thomas Graham McLaughlin, On the intersection of

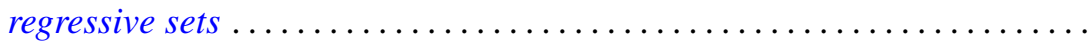

Murray Bell, John Norman Ginsburg and R. Grant Woods, Cardinal inequalities for topological spaces involving the weak Lindelof

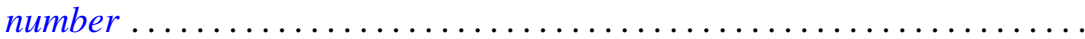

Laurence Richard Boxer, The space of ANRs of a closed surface ............

Zvonko Cerin, Homotopy properties of locally compact spaces at

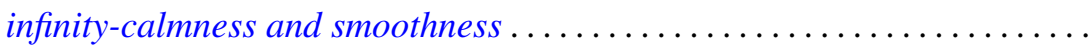

Isidor Fleischer and Ivo G. Rosenberg, The Galois connection between partial functions and relations..................................

John R. Giles, David Allan Gregory and Brailey Sims, Geometrical implications of upper semi-continuity of the duality mapping on a Banach

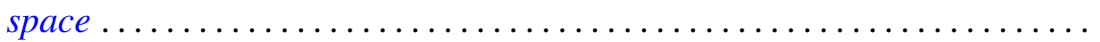

Troy Lee Hicks, Fixed-point theorems in locally convex spaces ............ Hugo Junghenn, Almost periodic functions on semidirect products of transformation semigroups ........................

Victor Kaftal, On the theory of compact operators in von Neumann algebras. II . . . .

Haynes Miller, A spectral sequence for the homology of an infinite

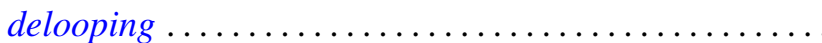

Sanford S. Miller, Petru T. Mocanu and Maxwell O. Reade, Starlike integral operators...

Stanley Stephen Page, Regular FPF rings ...............

Ghan Shyam Pandey, Multipliers for C, 1 summability of Fourier series ...

Shigeo Segawa, Bounded analytic functions on unbounded covering surfaces...

Steven Eugene Shreve, Probability measures and the C-sets of

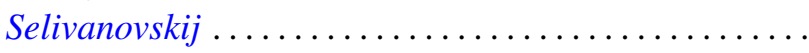

Tor Skjelbred, Combinatorial geometry and actions of compact Lie groups....

Alan Sloan, A note on exponentials of distributions.

Colin Eric Sutherland, Type analysis of the regular representation of a nonunimodular group.

Mark Phillip Thomas, Algebra homomorphisms and the functional

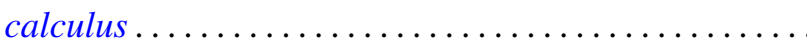

Sergio Eduardo Zarantonello, A representation of $H^{p}$-functions with

$0<p<\infty$. 